\title{
A Phase III, Randomized, Multi-Center, Double- Masked, Matched-Pairs, Active-Controlled Trial to Compare the Efficacy and Safety between Neuramis Deep and Restylane in the Correction of Nasolabial Folds
}

\author{
Changsik Pak ${ }^{1}$, Jihoon Park ${ }^{1}$, Jinmyung Hong ${ }^{1}$, Jaehoon Jeong ${ }^{1}$, Saik Bang ${ }^{2}$, Chan Yeong Heo ${ }^{1}$ \\ ${ }^{1}$ Department of Plastic and Reconstructive Surgery, Seoul National University Bundang Hospital, Seoul National University College of \\ Medicine, Seongnam; ${ }^{2}$ Department of Plastic and Reconstructive Surgery, Samsung Medical Center, Sungkyunkwan University School of \\ Medicine, Seoul, Korea
}

Background We conducted this clinical study to compare the efficacy and safety between Neuramis Deep and Restylane in the correction of nasolabial folds.

Methods In this phase III, randomized, multi-center, double-masked, matched-pairs, activecontrolled trial (ClinicalTrials.gov Identifier: NCT01585220), we evaluated a total of 67 subjects $(n=67)$. All the subjects underwent Neuramis Deep treatment on one side and Restylane on the contralateral side of the bilateral nasolabial folds at a ratio of 1:1. To compare the efficacy of Neuramis Deep and Restylane, we evaluated the Wrinkle Severity Rating Scale scores and those of the Global Aesthetic Improvement Scale. In addition, we compared the safety of Neuramis Deep and Restylane based on adverse events, physical examination, and clinical laboratory tests.

Results Neuramis Deep was not inferior in improving the nasolabial folds as compared with Restylane. In addition, there was no significant difference in the efficacy between Neuramis Deep and Restylane. There were no significant differences in safety parameters between Neuramis Deep and Restylane.

Conclusions In conclusion, our results indicate that Neuramis Deep may be a safe, effective material for improving the nasolabial folds. However, further studies are warranted to compare the tolerability of Neuramis Deep and Restylane based on histopathologic findings.

Keywords Hyaluronic acid / Nasolabial fold
Correspondence: Chan Yeong Heo Department of Plastic and Reconstructive Surgery, Seoul National University Bundang Hospital, Seoul National University College of Medicine, 82 Gumi-ro 173beon-gil, Bundang-gu, Seongnam 13620, Korea Tel: +82-31-787-7222

Fax: +82-31-787-4055

E-mail: lionheo@snu.ac.kr

The current study was financially supported by a grant of the Korea Healthcare Technology R\&D Project, Ministry of Health \& Welfare, Republic of Korea (A100054).

No potential conflict of interest relevant to this article was reported.

\section{INTRODUCTION}

Hyaluronic acid (HA) is a polyanionic mucopolysaccharide that is widely present in nature and extensively distributed in the con- nective tissues, including the skin and synovial fluid of the joints. It has high viscosity and hydrophilic properties, and is involved in many biological functions of the human body. However, it is promptly degraded by hyaluronidase, with a short half-life of 
approximately one day. Because its effects are not sustained, it cannot be effectively used for procedures such as anti-wrinkle treatments. To overcome this disadvantage, efforts have been made to develop crossed-linked HA fillers that are highly resistant to enzymatic degradation [1]. As high molecular weight compounds with excellent biocompatibility, they are currently used for clinical purposes such as soft tissue augmentation, antiarthritic therapy, wound healing, drug delivery, and tissue engineering.

For the development of HA with increased resorbability and sustained biocompatibility, diverse HA technologies have been used to modify or stabilize HA and to increase the product quality. It is the current trend to develop HA technologies for maximizing the cross-linking reaction while minimizing the use of the cross-linking reagent and the amount of residual cross-linker during the refining process. The chemical cross-linking of HA has been actively studied over the past two decades. This has led to the development of new cross-linking technologies such as photoreactive and ionic cross-linking. Various methods are used for cross-linking high molecular-weight HA; these include diepoxies, carbodiimides, aldehydes, divinyl sulfone, and photoreactive crosslinking.

Diverse materials have been used to develop dermal fillers for the past forty years. Since the 1980s, collagen fillers have been widely used for aesthetic purposes [2]. In addition, HA fillers have also been introduced and used clinically for approximately the past twenty years. In the early stages, HA fillers have been used mainly for nonaesthetic purposes, such as opthalmologic surgery, anti-arthritic therapy, and wound healing, rather than aesthetic ones. As it has become more widely used for aesthetic purposes in recent years, many advances have been made in the development of these fillers, leading to increased quality. In addition, it is generally known that HA fillers are stabilized through minimal chemical modifications. It is therefore probable that there may be improvement in resistance to enzymatic degradation in and biocompatibility with the dermis [3].

Neuramis Deep (Medytox, Seoul, Korea) is a monophasic, colorless, and transparent non-animal-derived stabilized HA (NASHA) filler (molecular weight: $1 \mathrm{MDa}$ ), and it was developed using stable hyaluronic acid and purification enhancement (SHAPE) cross-linking technology. Restylane (Q-Med, Uppsala, Sweden) is a biphasic NASHA filler (molecular weight: 1 $\mathrm{MDa}$ ) that was produced using a different bacterial fermentation system (Streptococcus equi).

Given the above background, we conducted this clinical study to compare the efficacy and safety of Neuramis Deep and Restylane in the correction of nasolabial folds (Table 1).

\begin{tabular}{|c|c|c|}
\hline Variable & Neuramis & Restylane \\
\hline Manufacturer & Medytox & Q-Med \\
\hline Hyaluronic acid molecular weight (Da) & $1.0 \times 10^{6}$ & $1.0 \times 10^{6}$ \\
\hline Hyaluronic origin & $\begin{array}{l}\text { Streptococcus } \\
\text { zooepidemicus }\end{array}$ & Streptococcus equi \\
\hline Cross-linker & BDDE & BDDE \\
\hline Cross-linking technology & SHAPE & NASHA \\
\hline Gel phase & Monophasic & Biphasic \\
\hline HA content (mg/mL) & 20 & 20 \\
\hline Storage modulus (Pa) & 120 & 300 \\
\hline Elasticity (\%) & 82.6 & 82.5 \\
\hline Modification (\%) & 8.5 & 0.7 \\
\hline
\end{tabular}

\section{METHODS}

\section{Study subjects}

The current study is a phase III, randomized, multicenter, double-masked, matched-pairs, active-controlled trial (ClinicalTrials.gov Identifier: NCT01585220), and it was conducted at two medical institutions during a period ranging from February 9 to October 30, 2012. To determine the sample size, the mean difference in the change of the Wrinkle Severity Rating Scale scores (WSRS) from baseline to week 24 between the study group (Neuramis) and control group (Restylane) was assumed to be 0 , and the standard deviation of the difference in the change was assumed to be 0.75 using the standard deviation of Restylane determined in a previous study [4]. The clinical interiority margin was also assumed to be 0.285 , half of 0.57 , which was the difference between groups (Restylane-Zyplast) also noted in a previous study [4]. Considering a $2.5 \%$ level of significance, a power of $80 \%$, and a $20 \%$ drop-out rate, the total target number of subjects was 69 . We recruited a total of 74 subjects at two medical institutions, but excluded 5 through baseline screening and selection criteria (Table 2). We therefore enrolled a total of 69 subjects in the current study, but two of them dropped out because they withdrew written informed consent or were found not to meet inclusion criteria. A total of 67 subjects $(n=67)$ completed the current study, all of whom submitted written informed consent. All the subjects underwent Neuramis Deep treatment on one side and Restylane on the contralateral side of the bilateral nasolabial folds at a ratio of 1:1. The current study was approved by the Institutional Review Board of each institution.

\section{Study materials}

Investigational material

Neuramis Deep was injected into the dermis using a 27-gauge 
Table 2. Inclusion and exclusion criteria

Inclusion and exclusion criteria
Inclusion criteria
1) Men and women, $30-70$ years old
2) Subjects desiring to correct a nasolabial fold and who have a Wrinkle Severity Rating Scale score of 3 or 4
3) Subjects with visually symmetric nasolabial folds
Exclusion criteria
1) Subjects taking anti-coagulant therapy (excluding low-dose aspirin; maximum, 300 mg/day dose) therapy within 2 weeks of the screening date
2) Subjects who had previously undergone treatment of the lower orbital rim for wrinkle correction (e.g., face lift, soft tissue augmentation, medium depth peels, dermal
photorejuvenation) within 6 months of the screening date
3) Subjects who had permanent implants for dermal augmentation (e.g., silicone) in the nasolabial fold area
4) Subjects who have a scar or skin lesion in the nasolabial fold area which could affect the discernment of treatment efficacy
5) Subjects having experienced anaphylaxis or a severe combined allergy, or an allergy to lidocaine or hyaluronic acid
6) Subjects with a history of keloid formation or hypertrophic scarring
7) Subjects who had other underlying systematic diseases

\section{Fig. 1. Schematic diagram of the study design}

At two weeks after the treatment, the subjects visited a study center and returned their diary. All the subjects visited a study center at 2, 8, 16, and 24 weeks. At each visit, except for the screening visit, they underwent clinical photography at the sites of the injection of the study material for the assessment of efficacy and safety. WSRS, Wrinkle Severity Rating Scale scores.

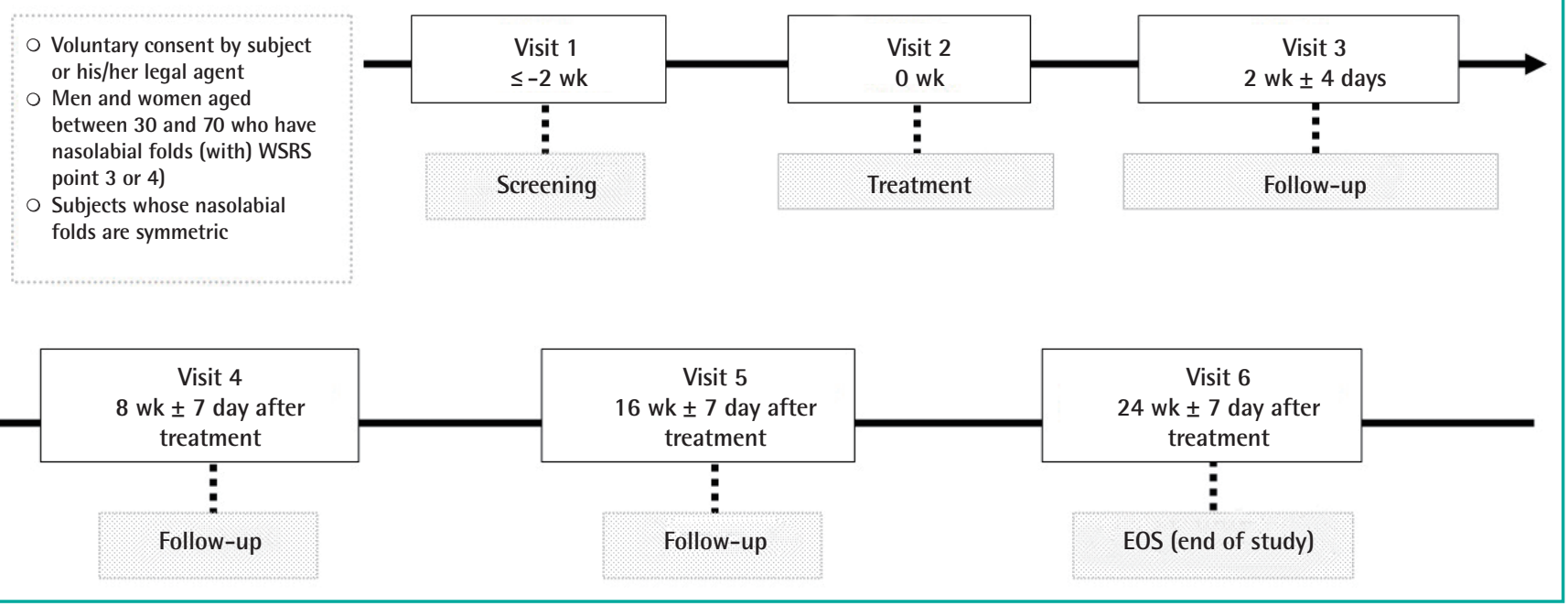

needle in the unilateral nasolabial folds. After insertion in the injection sites, the needle was retracted while the material was injected. To prevent the leakage of injection material, the injection was discontinued immediately before the needle was pulled out of the port. It is recommended that Neuramis Deep be injected at an optimal dose of $<1 \mathrm{~mL}$ (Table 2 ).

\section{Comparator}

Restylane was injected in the dermis using a 30-gauge needle in the contralateral nasolabial folds. After insertion into the sites of injection, the needle was retracted while it was injected. To prevent the leakage of injection material, the injection was discontinued immediately before the needle was pulled out of the port. It is recommended that Restylane be injected at an optimal dose of $<1 \mathrm{~mL}$ (Table 2).

\section{Study design}

Once enrolled in the current study, all the subjects were evaluated for their WSRS scores by study investigators prior to the injection of the study material. All the subjects underwent Neuramis Deep treatment on one side and Restylane on the contralateral side of the bilateral nasolabial folds at a ratio of 1:1. At a given study center, the subjects were treated and evaluated by the same investigators. Thus, attempts were made to minimize the bias due to the differences in treatment modalities and to consistently assess the efficacy throughout the study period. Study details were disclosed to the investigators who treated the subjects using the randomization chart, but those who assessed the outcomes were blinded. Thus, attempts were made to maintain the consistency and objectivity of assessment.

Moreover, the subjects were evaluated for the occurrence of adverse events (AEs) for 30 minutes immediately after receiving 
the treatment. They were given a diary and then instructed to record the appearance and disappearance of AEs for the following two weeks. At two weeks after the treatment, the subjects visited each study center and returned their diary. All the subjects visited each study center at 2, 8, 16, and 24 weeks. At each visit, except for the initial screening visit, they underwent clinical photography at the sites of the injection of the study material for the assessment of efficacy and safety (Fig. 1).

\section{Efficacy outcome measures}

Primary outcome measures

The primary outcome measure was non-inferiority of the efficacy of Neuramis Deep in improving the wrinkles as compared with Restylane based on changes in the WSRS scores on clinical photography at 24 weeks after the treatment of nasolabial fold as compared with baseline (Table 3 ).

\section{Secondary outcome measures}

(1) Changes in the WSRS scores at 2, 8, 16, and 24 weeks after the treatment as compared with baseline according to the judgement of the investigators performing follow-up physical examinations.

(2) Changes in the WSRS scores at 2, 8, and 16 weeks after the treatment as compared with baseline on the evaluation of the clinical photography by independent evaluators.

(3) The proportion of the subjects whose Global Aesthetic Improvement Scale (GAIS) scores were at least 1 point at 2, 8, 16, and 24 weeks after the treatment as compared with baseline according to the judgment of the investigators.

(4) The proportion of the subjects whose GAIS scores were at least 1 point at $2,8,16$, and 24 weeks after the treatment as compared with baseline according to the judgment of the subjects.

(5) The proportion of the subjects for whom the WSRS scores showed a change by at least 1 point at $2,8,16$, and 24 weeks after the treatment as compared with baseline according to the judgment of the investigators.

(6) The proportion of the subjects for whom the WSRS scores showed a change by at least 1 point at $2,8,16$, and 24 weeks after the treatment as compared with baseline on the evaluation of the clinical photography by the independent evaluators.

\section{Safety analysis}

\section{Adverse events}

We evaluated all the AEs that occurred in the subjects who submitted written informed consent, and these included the following: (1) The AEs that occurred only after the subjects submitted written informed consent. (2) The AEs that were aggravated after the subjects submitted written informed consent although they did occur before (Those AEs with previous treatment history were excluded).

We noted the number of cases of these AEs, and the proportion of the subjects who presented with AEs more than once. We also noted their severity. In noting the frequency of AEs, we analyzed the overall frequency of AEs, ADEs, and SAEs. Then, we compared these values between the two groups.

\section{Assessment scales}

The WSRS is widely used to grossly assess the severity of wrinkles and folds, including facial ones in particular, in clinical studies $[4,5]$. It was also used as the primary evaluation scale for the assessment of the efficacy of Restylane, and served as the comparison value in the current study, prior to its marketing approval by the US Food and Drug Administration. It has been estab-

\begin{tabular}{|c|c|c|c|c|}
\hline WSRS score & $\begin{array}{c}\text { Neuramis } \\
(\text { mean } \pm \text { SD) }\end{array}$ & $\begin{array}{c}\text { Restylane } \\
(\text { mean } \pm \text { SD) }\end{array}$ & $\begin{array}{l}\text { Difference } \\
(95 \% \mathrm{Cl})\end{array}$ & P-value \\
\hline \multicolumn{5}{|l|}{$\mathrm{ITT}(\mathrm{n}=68)$} \\
\hline Baseline & $2.85 \pm 0.71$ & $2.90 \pm 0.68$ & \multirow{4}{*}{$0.17(0.08-0.26)$} & \multirow[t]{2}{*}{$0.511^{\mathrm{a}}$} \\
\hline 24 weeks & $2.35 \pm 0.81$ & $2.56 \pm 0.78$ & & \\
\hline Change & $0.50 \pm 0.68$ & $0.34 \pm 0.65$ & & $<0.001^{a)}$ \\
\hline P-value & $<0.001^{\text {a) }}$ & $<0.001^{\text {a) }}$ & & \\
\hline \multicolumn{5}{|l|}{$P P(n=65)$} \\
\hline Baseline & $2.85 \pm 0.71$ & $2.91 \pm 0.69$ & \multirow{4}{*}{$0.18(0.09-0.27)$} & \multirow[t]{2}{*}{$0.419^{a)}$} \\
\hline 24 weeks & $2.33 \pm 0.80$ & $2.57 \pm 0.79$ & & \\
\hline Change & $0.50 \pm 0.66$ & $0.34 \pm 0.66$ & & $<0.001^{\text {a) }}$ \\
\hline P-value & $<0.001^{\text {a) }}$ & $<0.001^{\text {a) }}$ & & \\
\hline \multicolumn{5}{|c|}{$\begin{array}{l}\text { WSRS, Wrinkle Severity Rating Scale scores; SD, standard deviation; Difference, } \\
\text { study group-control group; Cl, confidence interval; ITT, intent-to-treat; Change, } \\
\text { baseline value-week } 24 \text { value; PP, per-protocol. } \\
\text { alWilcoxon signed rank test. }\end{array}$} \\
\hline
\end{tabular}

\section{Table 3 . The wrinkle severity rating scale}

\begin{tabular}{|c|c|}
\hline Point & Description \\
\hline 5 & $\begin{array}{l}\text { Extreme: extremely deep and long folds; } 2-4 \mathrm{~mm} \text {, visible } \mathrm{v} \text {-shaped fold when stretched; detrimental to appearance; unlikely to have satisfactory correction with } \\
\text { injectable implant alone. }\end{array}$ \\
\hline 4 & Severe: very long and deep; prominent facial feature; less than $2 \mathrm{~mm}$, visible fold when stretched. \\
\hline 3 & Moderate: moderately deep fold; clear facial feature visible at normal appearance, but not when stretched. Excellent correction expected. \\
\hline 2 & Mild: shallow, but visible fold with slight indentation; minor facial feature. \\
\hline 1 & Absent: no visible fold; continuous line. \\
\hline
\end{tabular}


lished as providing detailed information about the severity of wrinkles and folds. Finally, the WSRS scores were evaluated by the investigators and the independent photography evaluators.

The GAIS was used to assess the degree of satisfaction with treatment outcomes by the investigators and subjects based on the criteria shown in Tables 3 and 4.

We evaluated the treatment outcomes based on clinical photography, the WSRS scores, and the GAIS scores at 2, 8, 16, and 24 weeks after the treatment as compared with baseline.

\section{Data analysis}

\section{Statistical analysis}

In this study, statistical tests were conducted at significance level of 0.05 on both sides, unless specified otherwise. The primary efficacy criterion was the mean change in the WSRS at Week 24 from baseline as assessed by raters of photos. The $95 \%$ confidence interval of the mean of the treatment difference was calculated using the paired t-test, with non-inferiority declared if the interval was above the margin of -0.285 in both the full-analysis set and per-protocol (PP) populations. Continuous variables were summarized with descriptive statistics including the baseline value, each endpoint value, and the difference between the baseline and each endpoint. The changes were analyzed using the paired t-test or Wilcoxon's signed rank test. Categorical variables of local AEs were put into a shift table and analyzed using McNemar's test.

\section{Efficacy set}

In the current study, we performed the intent-to-treat (ITT) analysis for the assessment of the efficacy of treatment modalities. Additionally, we also performed the PP analysis.

ITT set: The ITT set comprised all the subjects who were given a randomization number after being enrolled in the current study. However, we excluded the following subjects: (1) The subjects who were enrolled in the current study although they did not meet inclusion/exclusion criteria at the screening visit. (2) The subjects who were enrolled in the current study but did not undergo treatment. (3) The subjects who were enrolled in the current study but did not undergo efficacy analysis.

PP set: The PP set comprised all the ITT subjects who completed the current study without seriously violating the study protocol. However, we excluded the following subjects: (1) The subjects who did not submit written informed consent. (2) The subjects who were not evaluated for efficacy at 24 weeks. (3) The subjects who underwent procedures or treatments that might affect the results of the efficacy analysis (including prohibited concomitant medications) during the study period.

\section{Safety set}

The safety analysis set comprised all the subjects who were enrolled in the current study and received the safety analysis after the treatment.

\section{RESULTS}

\section{Baseline characteristics}

In the current study, we enrolled a total of 69 subjects ( 68 women [98.55\%] and one man [1.45\%]). However, two of them were dropped out of the current study; one withdrew written informed consent, and the other did not meet inclusion/exclusion criteria. We therefore evaluated a total of 67 subjects who completed the current study throughout the study period. The mean age of the subjects was $48.96 \pm 8.90$ years.

The number of the subjects of the ITT set, the PP set, and the safety set were 68,65 , and 69 , respectively.

\section{Efficacy outcomes}

\section{Primary efficacy outcome measures}

In the ITT analysis, changes in the WSRS scores at 24 weeks as compared with baseline, which served as the primary efficacy outcome measures in the current study, were $0.50 \pm 0.66$ in the Neuramis Deep group and $0.34 \pm 0.65$ in the Restylane group. Therefore, the difference in the rate of the improvement between the two groups was 0.17 , for which the two-sided $95 \%$ interval was calculated as $(0.08,0.26)$. The lower limit of the one-sided $97.5 \%$ confidence interval was 0.08 , being greater than the maximum allowance of -0.285 based on which the clinical non-inferiority could be determined. These results indicate that Neuramis Deep is non-inferior to Restylane (Table 4).

\section{Secondary efficacy outcome measures}

In addition, there were significant differences in the mean WSRS on clinical photography between the two groups at 24 weeks as compared with baseline.

On the PP analysis, the lower limit of the one-sided $97.5 \%$ confidence interval was 0.09 . This also demonstrated that Neuramis Deep is non-inferior to Restylane. On the ITT, according to the judgment of the investigators, changes in the WSRS scores at 8 weeks as compared with baseline were $1.63 \pm 0.54$ in the Neuramis Deep group and $1.44 \pm 0.61$ in the Restylane group. These results indicate that there was a significant difference in the rate of improvement between the two groups $(\mathrm{P}=0.001)$. In addition, on the PP analysis, they were $1.62 \pm 0.55$ and $1.45 \pm 0.61$ in the corresponding order. These results indicate that there was a significant difference in the rate of improvement between the two groups $(\mathrm{P}=0.003)$. 


\section{Fig. 2. Secondary efficacy outcome of the study}

Changes in the Wrinkle Severity Rating Scale from baseline as evaluated by the investigators (Intent-to-Treat set). ${ }^{*} P<0.05$. These results indicate that there was a significant difference in the rate of improvement between the two groups $(P=0.001)$.

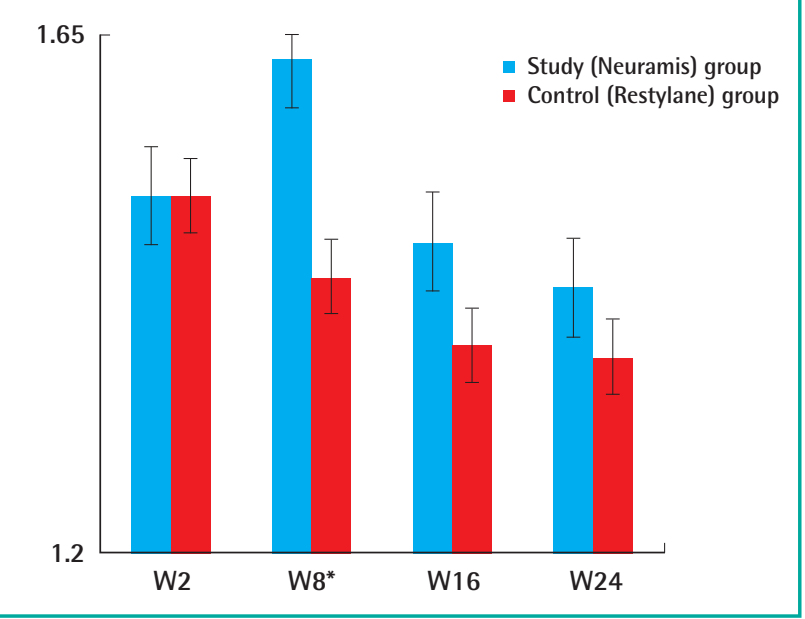

On clinical photography, there were changes in the WSRS scores at 2,8 , and 16 weeks as compared with baseline between the two groups in both the ITT and the PP analysis.

Finally, in both the ITT and the PP analysis, there were no significant differences in other efficacy variables between the two groups (Figs. 2-4).

\section{Safety outcomes}

\section{Adverse events}

Throughout the study period, a total of 76 cases of treatmentemergent AEs (TEAEs) occurred in 36 (52.17\%) of 69 subjects of the safety set. Of these, 10 cases were adverse drug events (ADEs) occurring in 5 (7.25\%) of 69 subjects of the safety set. In addition, one case of a serious adverse event (SAE) occurred in $1(1.45 \%)$ of 69 subjects of the safety set.

By the system organ class (SOC), there were 17 cases of infections and infestations occurring in 14 (20.29\%) of 69 subjects,

Fig. 3. Preoperative and postoperative photograph of a patient

(A) Preoperative. (B) Postoperative 24 weeks. Left nasolabial fold-Neuramis deep, right nasolabial fold-Restylane.
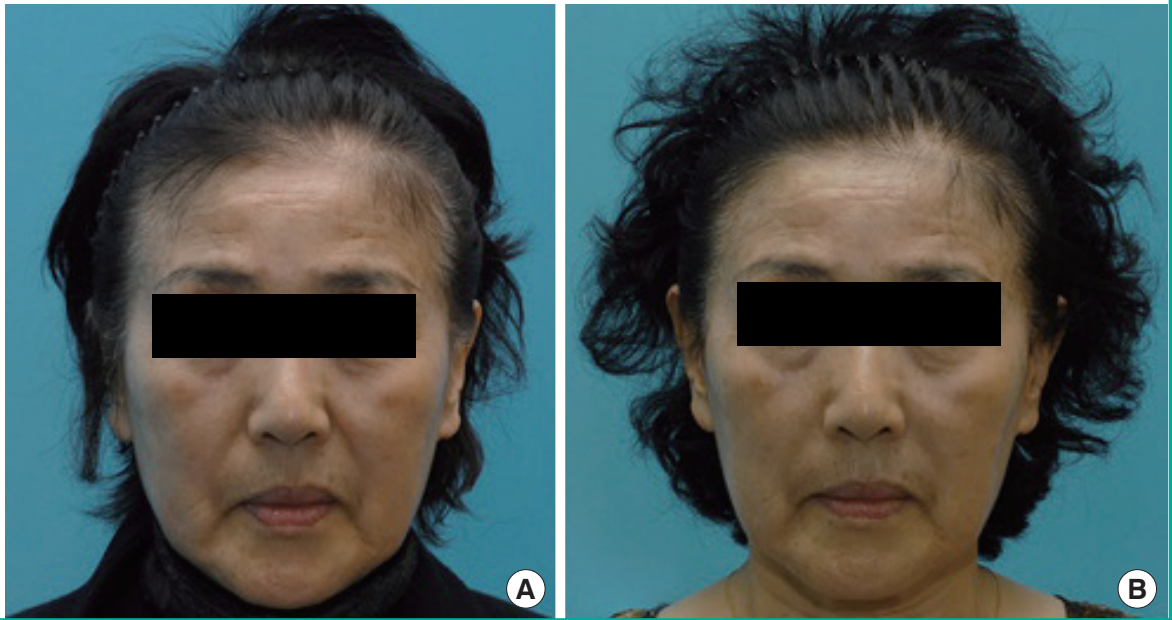

Fig. 4. Preoperative and postoperative photograph of another patient

(A) Preoperative. (B) Postoperative 24 weeks. Left nasolabial fold-Neuramis deep, right nasolabial fold-Restylane.
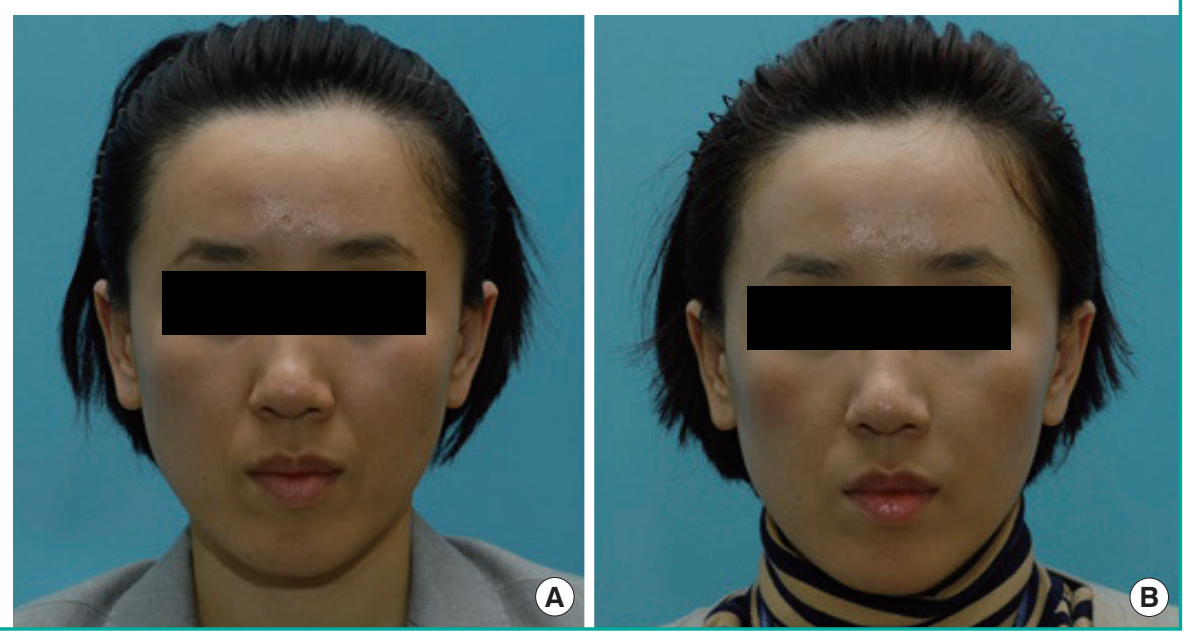
Table 5. Local adverse events

\begin{tabular}{|clccc|}
\hline & & Neuramis & Restylane & P-value \\
\hline Local adverse & $(-)$ & $11(15.94)$ & $10(14.49)$ & $0.655^{a)}$ \\
events & $(+)$ & $58(84.06)$ & $59(85.51)$ & \\
& Erythema & $21(30.43)$ & $21(30.43)$ & \\
& Pain & $32(46.38)$ & $32(46.38)$ & \\
& Edema & $43(62.32)$ & $41(59.42)$ & \\
& Pruritus & $29(42.03)$ & $29(42.03)$ & \\
& Discoloration & $1(1.45)$ & 0 & \\
Macule & 0 & $1(1.45)$ & \\
& Papule & 0 & $1(1.45)$ & \\
Retraction & $1(1.45)$ & $1(1.45)$ & \\
Bruise & $48(69.57)$ & $45(65.22)$ & \\
\hline
\end{tabular}

Values are presented as number (\%).

${ }^{a}$ McNemar's test.

11 cases of skin and subcutaneous tissue disorders occurring in 10 (14.49\%) of 69 subjects, and 10 cases of nervous system disorders occurring in 7 (10.14\%) of 69 subjects.

Treatment-emergent local AEs occurred at a frequency of $84.06 \%$ $(58 / 69)$ in the Neuramis Deep group and $85.51 \%(59 / 69)$ in the Restylane group. However, this difference was not statistically significant (Table 5). Moreover, the AEs at injection sites include bruising, occurring at a frequency of $69.57 \%(48 / 69)$, and edema, occurring at a frequency of $62.32 \%(43 / 69)$, in the Neuramis Deep group. They include bruising, occurring at a frequency of $65.22 \%$ (45/69), and edema, occurring at a frequency of $59.42 \%$ (41/69), in the Restylane group.

In the current study, there was one case of an SAE; a hemorrhoid occurred in one subject. This corresponded to an SAE for which in-hospital treatment was needed or the length of the hospital stay was prolonged. However, no subjects discontinued participation in the current study because of an SAE. Moreover, there was no causal relationship between the SAE and study material according to the judgment of the investigators. Finally, there were no unexpected ADEs or other notable AEs in the current study.

\section{Clinical laboratory parameters}

On clinical laboratory tests, basophil counts and serum potassium levels were significantly higher at 24 weeks as compared with baseline, but there were no significant changes in other clinical laboratory parameters. There were abnormal changes in WBC counts at 24 weeks as compared with baseline. However, there were no other clinical laboratory parameters showing abnormal changes at 24 weeks as compared with a normal baseline.

\section{DISCUSSION}

In the current study, we first compared the efficacy and safety between Neuramis Deep and Restylane in the correction of nasolabial folds. In many previous randomized, controlled studies, Restylane has served as the control group. To date, it has been widely used as an anti-wrinkle treatment by correcting subcutaneous depressions. Moreover, it has also been used for other cosmetic purposes. It is composed of stabilized HA, and it is a biodegradable, hydrophilic material. Once injected, it is gradually and completely absorbed into the body $[4,6]$. It is well known for its safety and excellent efficacy for volume augmentation. In the current study, we used it as the comparator because its composition, properties, biocompatibility, and mode of use are similar to those of Neuramis Deep. Neurami Deep has been developed using a unique cross-linking method (SHAPE technology), and it has different density zones in a single monophase solution. The SHAPE technology is referred to as a process where hyaluronic acid undergoes two-step cross-linking followed by enhanced purification. That is, a cross-linker binds to HA polymer in the primary cross-linking process. This is followed by a secondary cross-linking process where an unbound cross-linker binds to the HA polymer. Then, the unbound cross-linkers are removed through an enhanced purification process. Monophasic filler undergoes a two-step densification process. Monodensified filler undergoes only one cross-linking process, but polydensified filler undergoes a two-step cross-linking process after it has been added to the HA polymer following the primary crosslinking process. Neuramis Deep is a type of polydensified filler; it is synthesized through a two-step cross-linking process. Compared with monodensified gel, polydensified gel is less clumped and more evenly distributed. In contrast, monophasic gel is evenly distributed in the soft tissue, and it stretches out collagen fibers. It is therefore advantageous in being spread more evenly and restoring the skin volume defects more efficiently as compared with biphasic products [7].

It has been previously shown that the monophasic fillers contain collagen fibers that are present in a full layer of the reticular dermis, and they are separated by an abundant presence of HAlike amorphous material [8]. The HA fillers are prepared using different production technologies and are distributed differently in the dermis. The biphasic fillers such as Restylane form HA clumps, but the monophasic ones are distributed more evenly between the collagen fibers in the reticular dermis. HA fillers show variability in the time of absorption depending on the shape of the gel implant, the size of its particles, the status of aggregation, and the cross-linking status. Moreover, they are characterized by rapid absorption in such areas as the lip or nasolabial fold as compared with the cheek or chin. In particular, patients are initially satisfied with the injections of HA fillers when they feel that their wrinkles are temporarily filled because of injection-in- 
duced edema. With the degradation of HA fillers, however, patients become increasingly dissatisfied with treatment effects due to a shorter half-life. For these reasons, numerous fillers have been withdrawn from the market. Our results showed that the efficacy of Neuramis Deep was not inferior to that of Restylane. This indicates that the HA gel was distributed more evenly in the dermis. Park et al. reported that biphasic fillers have lower risks of overcorrection because of their localizing properties, whereas monophasic ones have a higher efficacy in restoring skin volume defects because they are distributed more evenly in the dermis [9]. Moreover, it has also been reported that monophasic fillers also have a higher efficacy in restoring skin volume defects arising from severe nasolabial folds or other facial wrinkles.

The ideal properties of monophasic fillers include excellent biocompatibility, the ease and convenience of injection, sustained cosmetic effects, and a lack of post-injection complications including pain. Both fillers had a favorable profile of safety and tolerability. They produced only mild AEs at injection sites, but some AEs are unavoidable because they are injected via a subcutaneous route. In the current study, there were delayed-onset reactions after the treatment with HA fillers. We assume, however, that these reactions would be of little or no clinical significance. Restylane has been used worldwide for several years, and it is close to the ideal filler. Our results indicate, however, that the efficacy and safety of Neuramis Deep are not inferior to those of Restylane in the correction of nasolabial folds.

To summarize, our results are as follows: (1) Neuramis Deep was not inferior in improving the nasolabial folds as compared with Restylane. In addition, there was no significant difference in the efficacy between Neuramis Deep and Restylane. (2) There were no significant differences in safety parameters between Neuramis Deep and Restylane.

In conclusion, our results indicate that Neuramis Deep might be a safe, effective material for improving the nasolabial folds. However, further studies are warranted to compare the tolerability between Neuramis Deep and Restylane based on histopathologic findings.

\section{REFERENCES}

1. Quirino MR, Neves AC, Campos MS, et al. Oral granuloma formation after injection of cosmetic filler. J Craniomaxillofac Surg 2012;40:e194-7.

2. Lindqvist $\mathrm{C}$, Tveten $\mathrm{S}$, Bondevik BE, et al. A randomized, evaluator-blind, multicenter comparison of the efficacy and tolerability of Perlane versus Zyplast in the correction of nasolabial folds. Plast Reconstr Surg 2005;115:282-9.

3. Matarasso SL, Carruthers JD, Jewell ML. Consensus recommendations for soft-tissue augmentation with nonanimal stabilized hyaluronic acid (Restylane). Plast Reconstr Surg 2006;117:3S-34S.

4. Narins RS, Brandt F, Leyden J, et al. A randomized, doubleblind, multicenter comparison of the efficacy and tolerability of Restylane versus Zyplast for the correction of nasolabial folds. Dermatol Surg 2003;29:588-95.

5. Carruthers A, Carey W, De Lorenzi C, et al. Randomized, double-blind comparison of the efficacy of two hyaluronic acid derivatives, restylane perlane and hylaform, in the treatment of nasolabial folds. Dermatol Surg 2005;31:1591-8.

6. Beer K. A randomized, evaluator-blinded comparison of efficacy of hyaluronic acid gel and avian-sourced hylan B plus gel for correction of nasolabial folds. Dermatol Surg 2007; 33:928-36.

7. Buntrock H, Reuther T, Prager W, et al. Efficacy, safety, and patient satisfaction of a monophasic cohesive polydensified matrix versus a biphasic nonanimal stabilized hyaluronic acid filler after single injection in nasolabial folds. Dermatol Surg 2013;39:1097-105.

8. Flynn TC, Sarazin D, Bezzola A, et al. Comparative histology of intradermal implantation of mono and biphasic hyaluronic acid fillers. Dermatol Surg 2011;37:637-43.

9. Park KY, Kim HK, Kim BJ. Comparative study of hyaluronic acid fillers by in vitro and in vivo testing. J Eur Acad Dermatol Venereol 2014;28:565-8. 